\title{
Engenharia Didática: Análises Preliminares e a Priori para a Noção dos Quaternions de Fibonacci.
}

\section{Didactical Engineering: Preliminary and a Priori Analysis for the Fibonacci's Quaternions.}

\author{
Francisco Regis Vieira Alves \\ Instituto Federal de Educação, Ciência e Tecnologia do Estado do Ceará. Programa de Pós-Graduação Stricto Sensu em Ensino de Ciências. CE, \\ Brasil. \\ Instituto Federal de Educação, Ciência e Tecnologia do Estado do Ceará. Programa de Pós-Graduação Stricto Sensu Mestrado Profissional em \\ Educação Profissional e Tecnológica. CE, Brasil. \\ E-mail:
}

\begin{abstract}
Resumo
O modelo matemático que, segundo os livros de História da Matemática, é atribuído, equivocadamente ao matemático Leonardo Pisano, preserva hodiernamente um vigor irrefreável de um progresso maior de evolução e de generalidade. Não obstante, um caráter de oblívio total pode ser observado no que concerne a uma maior divulgação e, também, melhor formação do professor de Matemática, concernentemente a determinados assuntos matemáticos específicos. Diante de tal entrave, o artigo atual apresenta a descrição das análises preliminares e análise a priori de uma Engenharia Didática, com o tema quaternions (generalizados) de Fibonacci. A relevância deste assunto se evidencia, na medida em que, o mesmo pode ser encontrado apenas em artigos científicos de Matemática que reproduzem propriedades formais cifradas. Assim, com arrimo da Teoria das Situações Didáticas - TSD, o trabalho apresenta, de modo pormenorizado, um planejamento para as fases dialéticas de ação, formulação, validação e institucionalização. Por fim, a atual proposta explora elementos que balizam sua eventual aplicação e experimentação em sala de aula, tendo como público alvo, professores de Matemática em formação inicial.
\end{abstract}

Palavras-chave: História da Matemática. Sequência de Fibonacci. Investigação Histórica.

\begin{abstract}
The mathematical model, according to the History of Mathematics books, is attributed wrongly to the mathematician Leonardo Pisano, in our times preserves an unstoppable force for further progress of evolution and generality. Nonetheless, a complete forgetfulness character can be seen in relation to greater disclosure and also improved training of the mathematics teacher, concernentemente to specific mathematical topics. Faced with this obstacle, the current article presents a description of the preliminary analysis and a priori analysis of a Didactic Engineering, with the theme quaternions (generalized) Fibonacci. The importance of this matter is evidenced, in so far as the same can be found only in scientific articles Mathematics reproducing encrypted formal properties. So with retaining the Theory of Didactic Situations - TSD, the work presents, in detail, a plan for the dialectics stages of action, formulation, validation and institutionalization. Finally, the current proposal explores elements that guide its possible application and experimentation in the classroom, with the target audience, mathematics teachers in initial training.
\end{abstract}

Keywords: Didactical Engineering. Fibonacci's Sequence. Quaternion of Fibonacci. Historical Investigation.

\section{Introdução}

Decidamente, a impressão transmitida pelos compendios de autores de livros de História da Matemática - HM, no que concerne a determinados tópicos ou assuntos particulares, não ultrapassa uma perspectiva superficial, limitada e, sobretudo, de um ponto de vista epistemológico, matemático e evolutivo, diríamos ainda incompleta. Nesse sentido, temos abordado em nossos trabalhos determinadas propriedades matemáticas generalizadas do modelo de Fibonacci (Alves, 2017) que, a despeito de seu marco histórico recorrentemente mencionado e atribuido a Leornardo Pisano (1170 - 1250), costuma ser desconsiderado e negligenciado por autores de livros de HM, no que concerne ao seu estádio matemático, epistemológico e evolutivo atual (Alves \& Borges Neto, 2011; Alves, 2015a; 2015b; 2016a; 2016b; 2016c; 2017).

Por um lado, assinalamos um carater compreensível deste fenômeno, posto que, determinadas variações e generalizações do modelo de Fibonacci podem ser averiguados em artigos acadêmicos recentes e que evidenciam, em maior ou em menor substância, o "estado de arte" sobre a matéria e tendem a envidar um estilo cifrado e sincopado do conhecimento matemático. Não obstante, apesar de referenciarmos uma vertente de investigação em Matemática pura e reuniu os estusiastas da sequência de Fibonacci, inaugurada na década de 60, com a fundação do periodico The Fibonacci Quartelly, não podemos nos furtar de envidar esforços com o escopo de proporcionar aos nossos professores de Matemática, em formação inicial, uma perspectiva evolutiva contígua do modelo de Fibonacci, que foi deflagrada com o modelo de nascimento de pares de coelhos mas, que, preserva um vigor científico irrefreável, constatado ainda nos dias atuais, diante da pesquisa de inúmeros especialistas. (Catarino, 2015; 2016; 2019).

Matematicamente, chamamos de Sequência Recursiva 
Linear Homogênea Recorrente e, de modo tradicional, conhecemos por Sequência de Fibonacci - SF e, denotamola por $f_{n+1}=f_{n}+f_{n-1}$, com $n=1,2,3,4,5 \ldots$.Na figura 1 deparamos uma figura ilustrativa de Gulberg (1997) que evidencia o viés de popularidade desta sequência, cuja figura mnemônica do crescimento infinito da população concorreu para a popularização do modelo correspondente de recorrência.

Figura 1 - Gullberg (1997, p. 286) descreve a sequência de coelhos imortais

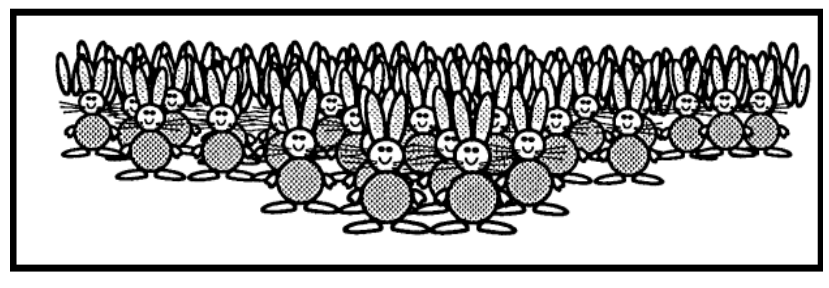

Isso posto, nos dois parágrafos anteriores, apontamos um entrave concernente ao entendimento de um processo epistemológico evolutivo de um modelo matemático ou sequência que preserva a atenção atual de pesquisadores em vários paises. Ademais, tendo em vista o interesse na obtenção e acúmulo de maiores conhecimentos didático-metodológicos sobre o assunto, escolheremos a Engenharia Didática ED, com o escopo de sistemátizar, planejar, predizer e, eventualmente, replicar o aparato teórico-conceitual balizado e estruturado a partir dos pressupostos e fundamentos da ED, em caráter de complementaridade com a Teoria das Situações Didaticas (Brousseau, 1986). Tal fundamentação deverá assegurar uma transposição didática (Chevallard, 1991) planejada e vinculada ao modelo matemático discutido, tendo em vista a inexistência de trabalhos acadêmicos semelhantes, que envolvam a discussão do tema ou assunto matemático aqui proposto.

Antes, porém, declaramos o seguinte elemento (questionamento) perquiridor que deverá consubstanciar, doravante, nossas escolhas: Como descrever uma abordagem para o ensino do modelo de Fibonacci, a fim proporcionar uma perspectiva e entendimento atual, não estático e hodierno do mesmo, no âmbito da formação (inicial) de professores?

Certamente que, a generalidade do questionamento anterior, tendo como escopo uma ação investigativa, não pode prescindir de perseguirmos um maior refinamento das intenções (e escolhas) ao longo do nosso percurso. Sendo assim, na seção subsequente, ao descrevermos alguns elementos de nossa metodologia ou design de pesquisa de natureza teórica, retomaremos o questionamento anterior, com a devida demarcação e pormenorização, do ponto de vista matemático e epistemológico do assunto que ensejamos apresentar para o leitor.

\section{Engenharia Didática: Alguns Elementos}

Recordamos que "o termo da Engenharia Didática designa um conjunto de sequências de classes concebidas, organizadas e articuladas no tempo, de maneira coerente por um professor-engenheiro, com o fim de realizar um projeto de aprendizagem para uma população determinada de alunos" (Douady, 1995b, p. 62). Não obstante, cabe explicarmos o emprego de determinados termos ou "metáforas" no excerto anterior.

Com efeito, os autores da vertente francesa costumam apoiar a descrição e a intenção de realizar um processo investigativo balizado pela Engenharia Didática - ED, com o escopo de realizar um projeto científico preciso e pormenorizado (Douady, 1995a; 1995b; 2008). Assim, de modo prosaico, todo o projeto ou a preparação deve ser pensada nos momentos de planejamento que antecedem a aula, propriamente dita, nos momentos de experimentação da aula, em que todo o aparato teórico conceitual é colocado em movimento e, finalmente, uma depuração dos dados examinados e da informações coligidas após ter sido encerrado o fenômeno aula, de sorte que, o conjunto das ações envolvendo o trinômio clássico (aluno - professor conhecimento matemático) devem concorrer para o acúmulo de conhecimentos didáticos-metodológicos sobre os assuntos de interesse da investigação, dos conhecimentos precisos e, também, particulares.

Não obstante, em alguns artigos científicos temos registrado o esforço investigativo no sentido de acumular conhecimentos didáticos e metodológicos acerca de determinados saberes oriundos no modelo de Fibonacci (Alves, 2015; 2016a; 2016b). Com amparo dessa perspectiva, propugnamos a existência de entrave um metodológico e epistemológico, tendo em vista o processo matemático evolutivo contíguo da Sequência Generalizada de Fibonacci, que pode ser apreciado em alguns artigos da década de 60, 70 e 80 (Harman, 1981; Hoggat, 1979; Waddill \& Sacks, 1967; Walton \& Horadam, 1961; 1963; 1967; 1974). Outrossim, não existem trabalhos semelhantes na literatura, com a discussão de alguns elementos teóricos de uma proposta teórica de ED, com o tema Quaternions de Fibonacci.

Dessa forma, com arrimo nos trabalhos pioneiros de Horadam $(1963 ;$ 1993), sabemos que um quaternion $Q$, com componentes reais $a_{0}, a_{1}, a_{2}, a_{3} \in I R$, e a base canônica $\{1, i, j, k\}$, é um número da forma $Q=a_{0} 1+a_{1} i+a_{2} j+a_{3} k, \quad$ com as seguintes regras operacionais ou regras $i^{2}=j^{2}=k^{2}=-1, i j k=1$. (Horadam, 1993).

Por outro lado, nosso maior interesse se origina da seguinte definição originada na década de 60 .

Definição 1: $\mathrm{O}$ número descrito por $Q_{n}=f_{n}+f_{n+1} i+f_{n+2} j+f_{n+3} k$ será chamado de n-ésimo quaternion de Fibonacci, e seus coeficientes satisfazem a relação de recorrência $f_{n+2}=f_{n+1}+f_{n}, n \geq 0$. (Horadam, 1963).

Ademais, da literatura, sabemos que seus valores 
iniciais são dados por $f_{1}=1, f_{2}=1$ e poderemos determinar o seguinte conjunto numérico que indicamos por $\left\{0,1,1,2,3,5,8,13,21,34,55, \ldots, f_{n}, \ldots\right\}$. Por outro lado, podemos determinar, também, ainda o um conjunto numérico ampliado, com valores inteiros não positivos (Abramovich, 2009; Alves, 2015a; 2015b; 2017).

$\left\{\ldots, f_{-n}, \ldots-55,34,-21,13,-8,5,-3,2,-1,1,0,1,1,2,3,5,8,13,21,34,55, \ldots, f_{n}, \ldots\right\}$

Assim, tendo em vista a definição 1, demarcamos a seguinte questão norteadora: Como conceber, descrever e estruturar uma abordagem metodológica para a noção de quaternion de Fibonacci, tendo em vista a exploração de propriedades matemáticas que evidenciam seu caráter epistemológico evolutivo?

Ora, a partir do questionamento anterior, assinalamos quatro elementos significativos para a presente pesquisa de caráter teórico: (i) a situação de oblívio dedicado por parte dos compêndios especializados de História da Matemática HM (Burton, 2007; Eves, 2004; Krantz, 2006; Singh, 1985), no sentido de explicar o estádio atual evolutivo do modelo generalizado de Fibonacci; (ii) a relevância da divulgação científica do modelo dos quaternions de Fibonacci, rotineiramente abordados apenas por artigos científicos e técnicos de uma área restrita de pesquisa em Matemática Pura; (iii) o entendimento, por parte do professor de Matemática em formação, do processo irrefreável evolutivo e epistemológico do modelo de Fibonacci, com origem em 1202, com Leonardo de Fibonacci, até os dias atuais, constatados por uma profusão de artigos científicos de Matemática Pura; (iv) a quase total inexistência de trabalhos acadêmicos que envolvam uma perspectiva de ensino dos Quaternions de Fibonacci.

E, após a indicação dos quatro itens anteriores, descrevemos uma problemática de nossa investigação não empírica. Destacamos que esta se caracteriza como "o conjunto de questões coordenadas que se coloca num determinado quadro teórico para esclarecer o problema levantado e os objetivos do estudo" (Almouloud, 2007, p. 169).

Isso posto, elegeremos os seguintes objetivos particulares: (a) discutir e pormenorizar propriedades matemáticas relacionadas com os quaternions de Fibonacci $Q_{n}=f_{n}+f_{n+1} i+f_{n+2} j+f_{n+3} k, \quad$ costumeiramente desconsideradas por autores de livros de HM; (b) descrever situações de ensino que proporcionem sua mediação em sala de aula; (c) descrever situações de ensino amparadas pela TSD envolvendo a noção de Quaternion de Fibonacci.

No próximo segmento, seguindo a tradição dos estudos da ED, trazemos uma análise matemático-epistemológica do nosso campo de interesse. Antes, porém, acentuamos que nosso percurso investigativo restringir-se-á ao estudo teórico da ED, isto é, ao estudo dos dados que independem dos elementos colhidos da realidade, originados a partir de uma eventual experimentação, mediante o exame das fases de análises a preliminares, análise a priori e concepção de situações. Dito de outra forma, tencionamos discutir apenas os momentos clássicos de análises preliminares, análise a priori envolvendo a concepção/descrição de situações didáticas (Almouloud, 2007) com o referido tema.

\section{Análises preliminares: sobre o conjunto dos quaternions de Fibonacci}

Uma condição sine qua non para o entendimento do processo epistemológico evolutivo da Matemática, em maior ou em menor substância, gira em torno do estabelecimento de definições matemáticas (Buffet, 2003). Ademais, parafraseando a história do nascimento do "ovo" e da "galinha", isto é, quem vem ou surge primeiro; todavia, no caso da Matemática, jamais conseguimos apreciar/confirmar, afirmar ou infirmar o estabelecimento de um teorema de modo que possamos prescindir, em sua demonstração, de uma definição matemática formal previamente elencada ou a priori definida.

Sendo assim, as definições matemáticas nos fornecem fortes e indissociáveis indícios do processo evolutivo matemático, tendo em vista que o trabalho matemático de sua construção, aceitação e estabelecimento confirma um viés estrutural e evolutivo em Matemática. Isto posto, para exemplificar. trazemos ao leitor as seguintes definições.

Definição 2: Chamaremos de números Gaussianos de Fibonacci, aos elementos descritos pela seguinte relação de recorrência $G f_{n}=G f_{n-1}+G f_{n-2}$, em que temos os valores inicias $G f_{0}=i, G f_{1}=1$. (Jordan, 1965).

Definição 3: Designaremos os Quaternions generalizados de Fibonacci de ordem 'n', por $P_{n}=h_{n}+h_{n+1} i+h_{n+2} j+h_{n+3} k$ , aonde $i^{2}=j^{2}=k^{2}=i j k=-1, i j=k=-j i, j k=i=-k j$, $k i=j=-i k$. (Iver, 1969).

Definição 4: Para ' $\mathrm{n}$ ' e ' $\mathrm{m}$ ' inteiros positivos, definiremos os números gaussianos de Fibonacci por $f_{n+m i}=\sum_{k=0}^{m}\left(\begin{array}{l}m \\ k\end{array}\right) \cdot i^{k} \cdot f_{n-k}$. (Bersenyi, 1977).

Definição5:Osnúmerosdaforma $G(n, m)$, devemsatisfazer

das seguintes condições bidimensionais de recorrência: $\{G(n+2, m)=G(n+1, m)+G(n, m)$

$\left\{\begin{array}{l}G(n, m+2)=G(n, m+1)+G(n, m)\end{array}\right.$, com as seguintes condições: $\quad G(0,0)=0, G(1,0)=1, G(0,1)=\mathrm{i}, G(1,1)=1+\mathrm{i}$.

(Harman, 1981).

Definição 6: Consideremos as seguintes relações tridimensionais.

$\left\{\begin{array}{l}G(h+2, l, m, n)=G(h+1, l, m, n)+G(h, l, m, n) \\ G(h, l+2, m, n)=G(h, l+1, m, n)+G(h, l, m, n) \\ G(h, l, m+2, n)=G(h+1, l, m+1, n)+G(h, l, m, n) \\ G(h, l, m, n+2)=G(h, l, m, n+1)+G(h, l, m, n)\end{array}\right.$ 


$$
\left\{\begin{array}{l}
G(0,0,0,0)=0, G(1,0,0,0)=1, G(0,1,0,0)=\mathrm{i} \\
G(0,0,1,0)=j, G(0,0,0,1)=\mathrm{k}, \\
G(1,1,0,0)=1+i, \ldots G(0,0,1,1)=\mathrm{j}+k \\
G(1,1,1,0)=1+i+k, \ldots G(0,1,1,1)=\mathrm{i}+\mathrm{j}+\mathrm{k} \\
\mathrm{G}(1,1,1,1)=1+i+j+k
\end{array}\right.
$$

, com as condições iniciais indicadas por. (Horadam, 1993).

Definição 7: $\quad$ O k-Quaternion dos k-numeros de Fibonacci, denotado por $D_{k, n}$, será definido por $D_{k, n}=f_{k, n}+f_{k, n+1} i+f_{k, n+2} j+f_{k, n+3} k$ aonde os termos presentes $f_{k, n}$ representam a sequência de k-números de Fibonacci (Ramírez, 2015).

Indubitavelmente, a partir de uma apreciação cuidadosa das (sete) definições matemáticas anteriores, o leitor poderá extrair um sentimento e entendimento do processo evolutivo de um modelo, porquanto, a despeito de vários teoremas e propriedades constatadas provisoriamente (Ramirez, 2015), bem como outras propriedades matemáticas questionadas (Polath \& Kesim, 2015), confirmado pelo debate científico entre os matemáticos profissionais, que ainda preserva seu vigor e o interesse atual da pesquisa em Matemática Pura (Tasci \& Yalcin, 2013).

Por outro lado, na próxima seção, trazemos ainda alguns dados que constituem elementos de uma análise epistemológica e histórica dos conteúdos que ensejamos perspectivar uma transposição didática (Chevallard, 1991) eficiente e metodologicamente planejada.

\section{As Repercussões na Pesquisa sobre os Quaternions de Fibonacci}

Brousseau (1989, p. 14) menciona que o matemático "não comunica seus resultados sob a forma que eles o encontra; ele os organiza, ele os fornece uma forma mais geral possível, ele desenvolve uma 'didática prática' que consiste em colocar o saber sobre forma comunicável, descontextualizada, despersonalizada e destemporalizada". Entretanto, no âmbito do ensino, deparamos um caráter antagonista (Margolinas, 1995, p. 343) ao fato indicado no excerto anterior. Com efeito, na frente do ensino, registramos um trabalho no sentido inverso, posto que o professor deverá recontextualizar, repersonalizar e modificar, de forma substancial, o saber científico, isto é, realizar uma transposição didática (Chevallard, 1991) eficiente, planejada e situada.

Com tal preocupação, vale recordar que, na literatura especializada encontramos indicações históricas (Conway \& Smith, 2003; Whittaker, 1945) sobre o estabelecimento final dos clássicos fundamentos necessários para a evolução dos quaternions (Hamilton, 1853) e, algum tempo depois, dos octônios, a partir da contribuição (e do insight) dos matemáticos irlandeses Sir William Rowan Hamilton (1805 - 1865) e John Thomas Graves (1806 - 1870) (Hanson, 2006, p. 9), perspectivados como uma extensão natural dos números complexos (Halici, 2012). No esteio do pensamento matemático evolutivo, podemos registrar, também, a pesquisa em torno dos sedênions (Soykan, 2019).

Com efeito, Hanson (2006, p. 8) recorda as inquietudes de Hamilton, quando proferiu a seguinte questão: "Se os quaternions generalizam os complexos, podem, então, eles próprios serem generalizados?". Em alguns dos trabalhos de Hamilton, a notação algébrica para vetores num espaço tridimensional, do tipo $x+i y+j z, i^{2}=j^{2}=-1$, em 1843, pode ser registrada (Whittaker, 1945, p. 95). Tal generalização pode estimular e aproximar os iniciantes no assunto, afim de compreender o caráter, por vezes, não fortuito da evolução matemática, de natureza epistemológica.

Por outro lado, Halici (2012a; 2012b) recorda que até a metade do século XX, se mostrava ser mínimo o seu uso, em comparação com outros métodos. Desse modo, constatamos um processo evolutivo do modelo da álgebra quadrimensional dos quaternions, bem como da álgebra octodimensional dos octônios que, ao passar do tempo, demonstrou uma região fractal como vários outros campos de investigação em Matemática Pura, sobretudo, no ãmbito das aplicações, como veremos logo mais adiante.

Mas, segundo o nosso interesse, perseguiremos um percurso evolutivo do modelo de Fibonacci, que segundo os autores de livros de HM, possui seu marco histórico com Leonardo Pisano, em 1202, apesar de que tais propriedade matemáticas de recorrência eram conhecidas pelos mercantilistas indianos, séculos e séculos antes (Singh, 2014) e podemos identificar outras imprecisões históricas relacionadas (Alves, 2017).

Ademais, Koshy (2007, p. 6) busca ainda esclarecer que:

[...] no Colégio Raj Narain em Bihar, na Índia, os números de Fibonacci e a sua formulação recursiva era conhecida na Índia, há vários séculos, proposto por meio de um problema por Virahanka (entre 600 e 800 A.D.), Gopala (depois de 1135 A.D.) e Hemacandra (em torno de 1150 A.D.). De fato, os números de Fibonacci foram estabelecidos, como fórmula, por Narayana Pandita (em 1356 A.D.).

Na figura 2, Conway \& Smiti (2003) fornecem uma representação $2 \mathrm{D}$ que serviu como modelo de representação para a forma complexa dos números de Fibonacci.

Figura 2 - Conway \& Smith (2003, p. 15) descrevem geometricamente um conjunto que impulsionou o interesse por representações no plano do modelo de Fibonacci

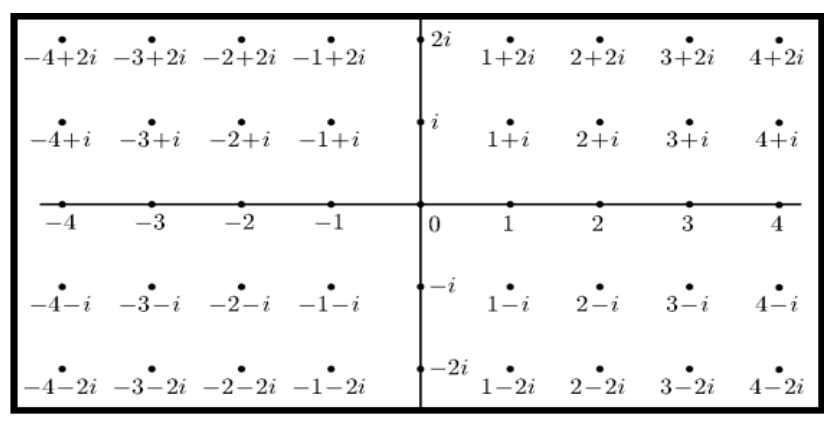

Por outro lado, passados alguns séculos, registramos uma mudança de perspectiva, com o intento de alguns matemáticos 
em estudar propriedades dos números de Fibonacci no plano. Dessa forma, na figura 2, divisamos uma representação dos inteiros gaussianos e, de modo particular no mesmo conjunto, Horadam (1963), Jordan (1965) e Berzsenyi (1975; 1977) se interessaram por um subconjunto que gozava da propriedade de recorrência de segunda ordem (ver definições 2 e 3). Logo em seguida, constatamos que a pesquisa evoluiu, no sentido de adaptar a ideia do modelo dos quaternions (Iakin, 1977; 1981) ao conjunto de Fibonacci e, ainda, pelo acréscimo das relações de recorrência (Horadam, 1993; Swamy, 1973).

Apesar de não tencionarmos maior aprofundamento no assunto, todavia, indicamos ao leitor um exame dos trabalhos e artigos científicos sobre os bicomplexos de Fibonacci, inspirados nos trabalhos pioneiros do matemático italiano Corrado Segre $(1863$ - 1924) ou, ainda, sobre a forma dual dos quaternions de Fibonacci (Nurkan \& Güven, 2015) e, também, da forma matemática generalizada, nominada como quaternions complexos de Fibonacci (Halici, 2013).

Com origem no contexto anterior, dois elementos devem ser evidenciados na etapa seguinte, de acordo com Almouloud (2007, p. 172), a saber: (i) estudo da organização matemática; (ii) análise didática do objeto matemático escolhido. Não obstante, como mencionamos nos parágrafos predecessores, no que concerne ao item (ii), um entendimento do ensino atual do assunto assumirá um viés restritivo, posto que, registramos o caráter de vacuidade de investigações de natureza semelhante ao tema desenvolvido ao longo do trabalho. Por sua vez, o componente evolutivo das definições matemáticas, em nosso caso particular, derivadas da sequência numérica e recursiva de Fibonacci, concorre para a compreensão da organização matemática e o devido acréscimo de uma cultura matemática por parte de professores de Matemática.

Nas quatro situações-problemas que abordaremos na próxima seção, assumiremos os pressupostos e a sistemática prevista pela ED. Ademais, tendo em vista a consideração de uma perspectiva de influência de caráter metodológico, assinalaremos a dialética dos momentos de ação, formulação, validação (Brousseau, 1986a) e a fase de institucionalização (Almouloud, 2007, p. 23), prevista em torno das modificações necessárias envolvendo as interações originadas do trinômio clássico (estudantes - professor - saber matemático).

\section{Analise a priori: concepção de situações didáticas}

A terminologia Engenharia Didática - ED foi usada para designar/envolver um modus operandi de investigação ou ainda como "uma metodologia para a análise de situações didáticas" (Robinet, 1983, p. 2). Dessa forma, com amparo numa mediação afetada por seus fundamentos e a tradição do uso, em caráter de complementaridade, de outras teorias que devem balizar uma eventual experimentação, acentuaremos as características a serem evidenciadas nas fases dialéticas previstas pela TSD, bem como uma mediação afetada por um conjunto de práticas discutidas sob a perspectiva da Didática da Matemática (Brousseau, 1986; 1988; 1998).

Sem mais delongas, apresentamos nossa primeira situação problema.

Situação problema I: No trabalho de Brousseau (1965) encontramos a seguinte identidade $f_{-n}=(-1)^{n+1} \cdot f_{n}, n \geq 0$. Tal relação possibilita a extensão da sequência de Fibonacci ao campo (de índices) inteiros registrada na literatura científica desde os anos 60. Decida se podemos determinar um comportamento semelhante no caso dos quaternions de Fibonacci, segundo a definição 1 .

Comentários: A propriedade que deve ser explorada no enunciado passou a ser relatada na literatura científica somente a partir dos anos 60 (Brother, 1965; Brousseau, 1971; Hoggat \& Vernner, 1969). Todavia, nos estudos interessados pelos quaternions e pelos quaternions de Fibonacci, observamos a constatação pelo interesse crescente décadas depois. Dessa forma, a primeira situação visa despertar a percepção do estudante de um processo que requereu algumas décadas, até ser concebido e discutido por meio da comunidade de matemáticos profissionais.

Situação de ação: “A constituição do sentido, tal como entendemos, implica numa interação constante dos alunos com situações problemáticas, interações dialéticas (caso o sujeito antecipe, finalize suas ações) [...]” (Brousseau, 1998, p. 117). Com origem na propriedade indicada, os estudantes devem testar/verificar alguns valores numéricos particulares. Assim, podem observar os casos

$$
\begin{array}{r}
Q_{0}=f_{0}+f_{1} i+f_{2} j+f_{3} k=0+i+j+2 k \\
Q_{1}=f_{1}+f_{2} i+f_{3} j+f_{4} k=1+i+2 j+3 k \\
Q_{2}=f_{2}+f_{3} i+f_{4} j+f_{5} k=1+2 i+3 j+5 k \\
Q_{3}=f_{3}+f_{4} i+ \\
+f_{5} j+f_{6} k=2+3 i+5 j+8 k
\end{array}
$$

Dessa forma, instigamos sua familiaridade numérica com um novo tipo de conjunto, cujos elementos devem ser indicados e originados pela relação $Q_{n}=f_{n}+f_{(n+1)} i+f_{(n+2)} j+f_{(n+3)} k$.

Situaçãodeformulação: Nafaseatual,oaumentoeaexigência depropriedades comosistemanotacional setornaindispensável. Assim, os estudantes devem ser estimulados a avaliar $Q_{-1}=f_{-1}+f_{0} i+f_{1} j+f_{2} k=(-1)^{1+1} f_{1}+(-1)^{0+1} f_{0} i+(-1)^{1+1} f_{1} j+(-1)^{2+1} f_{2} k=$

$$
1+0 i+j-k
$$

$$
+(-1)^{1+1} f_{1} i+(-1)^{0+1} f_{0} j+(-1)^{1+1} f_{1} k=-1+i-0 j+k
$$

Dessa forma, para alguns casos particulares, os estudantes devem explorar relações particulares do tipo $Q_{-6}=(-1)^{7}\left(f_{6}-f_{7} i+f_{8} j-f_{9} \mathrm{k}\right) \quad$ ou ainda $Q_{-10}=(-1)^{11}\left(f_{10}-f_{11} i+f_{12} j-f_{13} \mathrm{k}\right)$, etc, com arrimo da relação indicada no enunciado por $f_{n}=(-1) \cdot f_{n}, n \geq 0$.

Situação de validação: Nessa fase, num contexto do “debate da certeza das asserções" (Almouloud, 2007, p. 40), os dados produzidos com origem nas interações dialéticas dos estudantes da fase anterior, com as informações e inferências 
empregadas afim de obter a certeza das relações estabelecidas. Dessa forma, com origem na propriedade indicada, eles devem determinar que $Q_{-n}=f_{-n}+f_{-(n+1)} i+f_{-(n+2)} j+f_{-(n+3)} k=$

\section{$\left.(-1)^{n+1} f_{n}+(-1)^{n+2} f_{(n+1)} i+(-1)^{n+3} f_{(n+2)} j+(-1)^{n+4} f_{(n+3)} k=(-1)^{n+1} f_{n}-(-1)^{n+1} f_{(n+1)} i+(-1)^{n+1} f_{(n+2)} j-(-1)^{n+1} f_{(n-3)}\right)^{k}$}

$=(-1)^{n+1}\left(f_{n}-f_{(n+1)} i+f_{(n+2)} j-f_{(n+3)} \mathrm{k}\right) . \quad$ Portanto, devem encontrar a seguinte expressão algébrica final indicada $Q_{-n}=(-1)^{n+1}\left(f_{n}-f_{(n+1)} i+f_{(n+2)} j-f_{(n+3)} \mathrm{k}\right)$, para $n \geq 0$.

A relação anterior, permitirá o processo de extensão dos quaternions $\left\{Q_{-n}\right\}_{n \geq 0}$, com índices inteiros quaisquer, semelhante ao caso da Sequência de Fibonacci (Alves \& Borges Neto, 2011; Thongmoon, 2009). Isso posto, os elementos essenciais devem ascender ao status de patrimônio cultural matemático do grupo de estudantes e o professor. Deflagraremos, pois, a última fase da mediação.

Situação de institucionalização: Ora, o conhecimento matemático que o expert deverá convencionar ou fixar (Artigue, 1984, p. 8), seguindo os rituais acadêmicos, indicando o estatuto cognitivo de um novo saber, rico em relações conceituais. Dessa forma, deve ser apresentado o seguinte teorema que, constitui um caso particular o corolário indicado por Halici (2012).

Teorema 1: Os quaternios de Fibonacci definidos para índices inteiros quaisquer, será descrito por $Q_{-n}=(-1)^{n+1}\left(f_{n}-f_{(n+1)} i+f_{(n+2)} j-f_{(n+3)} \mathrm{k}\right), \quad$ com $n \geq 0$.

Vale observar que uma via mais eficiente de determinar o comportamento do quaternion $Q_{-n}$, se evidencia pelo uso da fórmula variante de Fibonacci que deve ser o assunto da próxima situação. Outrossim, em nossos trabalhos (Alves, 2016c), podemos apreciar a reação dos estudantes, de modo surpreso, com o estudo do modelo de Fibonacci extendido para índices inteiros quaisquer, que temos indicado por $f_{-n}$ , com $n \geq 0$.

Situação problema II: A fórmula explicita dos númerros de Fibonacci é conhecida na literatura por $f_{n}=\frac{\alpha-\beta}{\alpha-\beta}$ que, de formula de Binnet, ou de Moivre ou de Lamé (Alves, 2017), vários outros matemáticos concorreram para o seu irrefutável estabelecimento, como matemático francês Jacques Philippe Marie Binet (1786 - 1856), todavia, outros matemáticos produziram um resultado semelhante de modo independente. Diante dessa propriedade, decida se podemos extrair propriedade semelhante para o caso dos quaternions de Fibonacci.

Comentários: Decididamente, na literatura especializada, a fórmula $f_{n}=\frac{\alpha^{n}-\beta^{n}}{\alpha-\beta}$ ficou conhecida como fórmula de Binnet. Os alunos devem ser familiarizados que a equação característica da relação de recorrência $f_{n+2}=f_{n+1}+f_{n}$ deve determinar a equação $x^{2}-x-1=0$, donde inferimos as raízes $\alpha=\frac{1+\sqrt{5}}{2}, \beta=\frac{1-\sqrt{5}}{2}$.

Situação de ação: Os estudantes devem ser instigados na exploração de propriedades extraídas da fórmula $f_{n}=\frac{\alpha^{n}-\beta^{n}}{\alpha-\beta}$. Assim, com origem na equação $x^{2}-x-1=0$ e por indưção matemática, podemos encontrar que $\alpha^{n}=\alpha \cdot f_{n}+f_{n-1}$ e $\beta^{n}=\beta \cdot f_{n}+f_{n-1}$. A partir de um pensamento analógico, vemos $\alpha \cdot Q_{n}+Q_{n-1}=\alpha\left(f_{n}+i \cdot f_{n+1}\right.$

$\left.+j \cdot f_{n+2}+k \cdot f_{n+3}\right)+\left(f_{n-1}+i \cdot f_{n}+j \cdot f_{n+1}+k \cdot f_{n+2}\right)=\left(\alpha \cdot f_{n}+f_{n-1}\right)+i\left(\alpha \cdot f_{n+1}+f_{n}\right)+j\left(\alpha \cdot f_{n+2}+f_{n+1}\right)$
$+k\left(\alpha \cdot f_{n+3}+f_{n+2}\right)=\alpha^{n}+i \cdot \alpha^{n+1}+j \cdot \alpha^{n+2}+k \cdot \alpha^{n+3}=\alpha^{n} \cdot\left(1+i \alpha+j \alpha^{2}+k \alpha^{3}\right)=\alpha^{n} \cdot \alpha$
E, de modo similar, podem ver $\beta \cdot Q_{n}+Q_{n-1}=\beta\left(f_{n}+i \cdot f_{n+1}+j \cdot f_{n+2}+k \cdot f_{n+3}\right)+\left(f_{n-1}+i \cdot f_{n}+j \cdot f_{n+1}+k \cdot f_{n+2}\right)$ $=\left(\beta \cdot f_{n}+f_{n-1}\right)+i \cdot\left(\beta \cdot f_{n+1}+f_{n}\right)+j \cdot\left(\beta \cdot f_{n+2}+f_{n+1}\right)+k \cdot\left(\beta \cdot f_{n+3}+f_{n+2}\right)=$ $=\beta^{n}+i \cdot \beta^{n+1}+j \cdot \beta^{n+2}+k \cdot \beta^{n+3}=\beta^{n} \cdot\left(1+i \beta+j \beta^{2}+k \beta^{3}\right)=\beta^{n} \cdot \bar{\beta} \therefore \beta \cdot Q_{n}+Q_{n-1}=\beta^{n} \cdot \bar{\beta}$

Situação de formulação: Segundo Almouloud (2007, p. 38), uma das características dessa fase reside em "criar um modelo explícito que pode ser formulado com sinais e regras comuns, já conhecidas ou novas". Agora, seguindo ainda, por analogia o modelodafórmuladeBinnet, depossedasidentidades anteriores $\alpha^{n}=\alpha \cdot f_{n}+f_{n-1}$ e $\beta^{n}=\beta \cdot f_{n}+f_{n-1}$, podem determinar $\beta^{n}-\alpha^{n}=\beta \cdot f_{n}^{n}+f_{n-1}-\alpha \cdot f_{n}-f_{n-1}=\beta \cdot f_{n}-\alpha \cdot f_{n}=(\beta-\alpha) f_{n}$ - Assim, desde que, na fase anterior, as igualdades $\alpha \cdot Q_{n}+Q_{n-1}=\alpha^{n} \cdot \bar{\alpha} \quad$ e $\beta \cdot Q_{n}+Q_{n-1}=\beta^{n} \cdot \bar{\beta} \quad$ e, assim, empregar um argumento semelhante, bastando avaliar a seguinte diferença $\alpha \cdot Q_{n}+Q_{n-1}-\beta \cdot Q_{n}-Q_{\pi-1}=\alpha^{n} \cdot \bar{\alpha}-\beta^{n} \cdot \bar{\beta}$ . Portanto, vemos que $(\alpha-\beta) \cdot Q_{n}=\alpha^{n} \cdot \alpha-\beta^{n} \cdot \beta$.

Situação de validação: Almouloud (2007, p. 39) explica que "é a etapa na qual o aprendiz deve mostrar a validade do modelo por ele criado, submetendo a mensagem matemática (modelo de situação) ao julgamento de um interlocutor". Observamos que, nas fases anteriores, devem ser identificados os elementos principais que concorrem para a determinação da fórmula de Binnet para os quaternions.

Mas, com origem nos argumentos da fase anterior, encontramos $\alpha^{n} \stackrel{+}{\alpha}-\beta^{n}$ expressão $(\alpha-\beta) \cdot Q_{n}=\alpha^{n} \cdot \bar{\alpha}-\beta^{n} \cdot \bar{\beta} \therefore Q_{n}=\frac{\alpha^{n} \cdot \stackrel{\alpha}{\alpha}-\beta^{n} \cdot \beta}{(\alpha-\beta)}$

Que pode ser considerada como demonstração. Ademais, vejamos outra forma dedutiva do mesmo resultado ensejado no enunciado da situação II.

Demonstração 2:

$Q_{n}=f_{n}+i \cdot f_{n+1}+j \cdot f_{n+2}+k \cdot f_{n+3}=\left(\frac{\alpha^{n}-\beta^{n}}{\alpha-\beta}\right)+i \cdot\left(\frac{\alpha^{n+1}-\beta^{n+1}}{\alpha-\beta}\right)+$ $+j \cdot\left(\frac{\alpha^{n+2}-\beta^{n+2}}{\alpha-\beta}\right)+k \cdot\left(\frac{\alpha^{n+3}-\beta^{n+3}}{\alpha-\beta}\right)=\frac{\alpha^{n}\left(1+i \alpha+j \alpha^{2}+k \alpha^{3}\right)}{\alpha-\beta}-\frac{\beta^{n}\left(1+i \beta+j \beta^{2}+k \beta^{3}\right)}{\alpha-\beta}=$ $=\frac{\alpha^{n} \cdot \bar{\alpha}}{\alpha-\beta}-\frac{\beta^{n} \cdot \bar{\beta}}{\alpha-\beta}$, aonde

$\bar{\alpha}=1+i \alpha+j \alpha^{2}+k \alpha^{3}, \bar{\beta}=1+i \beta+j \beta^{2}+k \beta^{3}$

. Portanto, vemos que $Q_{n}=\frac{\alpha^{n} \cdot \bar{\alpha}-\beta^{n} \cdot \bar{\beta}}{\alpha-\beta}, n \geq 0$. Agora, com a ideia da situação anterior, podemos ver ainda que 
$Q_{-n}=f_{-n}+i \cdot f_{-(n+1)}+j \cdot f_{-(n+2)}+k \cdot f_{-(n+3)}=\left(\frac{\alpha^{-n}-\beta^{-n}}{\alpha-\beta}\right)+i \cdot\left(\frac{\alpha^{-(n+1)}-\beta^{-(n+1)}}{\alpha-\beta}\right)+$

$+j \cdot\left(\frac{\alpha^{-(n+2)}-\beta^{-(n+2)}}{\alpha-\beta}\right)+k \cdot\left(\frac{\alpha^{-(n+3)}-\beta^{-(n+3)}}{\alpha-\beta}\right)=\frac{\alpha^{-n}\left(1-i \alpha+j \alpha^{2}-k \alpha^{3}\right)}{\alpha-\beta}-\frac{\beta^{-n}\left(1-i \beta+j \beta^{2}-k \beta^{3}\right)}{\alpha-\beta}=$

$=\frac{\alpha^{n} \cdot \overline{\bar{\alpha}}}{\alpha-\beta}-\frac{\beta^{n} \cdot \overline{\bar{\beta}}}{\alpha-\beta}$

aonde $\quad \overline{\bar{\alpha}}=1-i \alpha+j \alpha^{2}-k \alpha^{3}, \overline{\bar{\beta}}=1-i \beta+j \beta^{2}-k \beta^{3} . \quad \mathrm{E}$,

dessa forma, devem estabelecer que $Q_{n}=\frac{\alpha^{n} \cdot \bar{\alpha}-\beta^{n} \cdot \bar{\beta}}{\alpha-\beta}, n \geq 0$ e também . $Q_{-n}=\frac{\alpha^{n} \cdot \overline{\bar{\alpha}}-\beta^{n} \cdot \overline{\bar{\beta}}}{\alpha-\beta}, n \geq 0$

Finalmente, podemos comparar a regularidade e invariância de determinados elementos atinentes aos dois modelos. Com efeito, no caso da sequência extendida de Fibonacci, temos $f_{-n}=(-1)^{n+1} f_{n}=(-1)^{n-1} f_{n}, n \geq 0$ - Todavia, os estudantes não devem confirmar a mesma

$$
Q_{n}=\left\{\begin{array}{l}
\frac{\alpha^{n} \cdot \bar{\alpha}-\beta^{n} \cdot \bar{\beta}}{\alpha-\beta}, n \geq 0 \\
\frac{\alpha^{n} \cdot \bar{\alpha}-\beta^{n} \cdot \overline{\bar{\beta}}}{\alpha-\beta}, n<0
\end{array}\right.
$$

propriedade, visto que . Dito de outra forma, a igualdade $Q_{-n}=(-1)^{n+1} Q_{n}=(-1)^{n-1} Q_{n}$ pode ser infirmada pelo grupo!

Situação de institucionalização: Brousseau (1986, p. 342) comenta que "a produção no ensino de conhecimento matemático demanda um esforço de transformação de um conhecimento em saber matemático [...]". Assim, ele indica que a epistemologia do professor atuará no sentido de não personalização e não contextualização, e que buscará eliminar os traços históricos que determinaram sua aparição.

Isso posto, uma apreciação não isolada de artigos científicos sobre a matéria deve inspirar o entendimento local e situado do grupo (professor e estudantes) acerca do processo matemático evolutivo e das propriedades algébricas invariantes que se manifestam na Sequência de Fibonacci e na classe dos Quaternions de Fibonacci.

Por outro lado, a distinção de Brousseau (1986) é clara, no sentido de pontuar um conhecimento mobilizado, localizado e particular, pertencente a um grupo de alunos e, gradualmente, receberá status de maior generalidade, pois, enquadrado num corpus teórico formal, cujo viés estruturalista se mostra evidenciado e distinguido na academia. Diante dessa perspectiva, afim acentuar o viés de um conhecimento em constante evolução, o professor deverá envidar esforços, no sentido de fazer aderir ao patrimônio coletivo do grupo, um teorema recentemente distinguido na pesquisa matemática, que enunciaremos como segue.

Teorema 2: Para os quaternions de Fibonacci, vale a seguinte forma variante da relação de Binnet, indicada por $Q_{n}=\frac{\alpha^{n} \cdot \bar{\alpha}-\beta^{n} \cdot \bar{\beta}}{\alpha-\beta}, n \geq 0$. (Akyigit; Kösal \& Tosun, 2014).

Situação problema III: O problema da produção dos coelhos, propugnado por Leonardo de Fibonacci, em 12002, pode ser traduzido em símbolos atuais pela equação $f_{n+2}=f_{n+1}+f_{n}$, e as condições $f_{1}=1, f_{2}=1$. Do ponto de vista matemático, lidamos com uma sequência de segunda ordem. Decida se os quaternions de Fibonacci gozam de propriedade semelhante, isto é, podemos contar com a relação de recursividade.

Comentários: A característica marcante da relação de Leonardo Pisano diz respeito ao seu grau de dependência dos dois termos imediatos antecessores. Assim, os alunos devem ser instigados a descobrir propriedade semelhante no conjunto dos quanternions de Fibonacci.

Situação de ação: Com origem nos valores particulares estabelecidos na situação I, os alunos devem observar que $Q_{0}+Q_{1}=(0+i+j+2 k)+(1+i+2 j+3 k)=1+2 i+3 j+5 k$

E, pelo mesmo argumento, devem determinar que $Q_{1}+Q_{2}=(1+i+2 j+3 k)+(1+2 i+3 j+5 k)=$

$=2+3 i+5 j+8 k$. E, ainda, mais uma vez que $Q_{2}+Q_{3}=(1+2 i+3 j+5 k)+(2+i 3+j 5+k 8)=$

$=3+5 i+8 j+11 k$. Por outro lado, em todos os casos anteriores, devem observar que $Q_{0}+Q_{1}=Q_{2}$, $Q_{1}+Q_{2}=Q_{3}, Q_{2}+Q_{3}=Q_{4}$ e, assim, devem ser instigados na obtenção das identidades subsequentes, vinculadas ao caráter de recursividade (de segunda ordem) dos quaternions de Fibonacci.

Situação de formulação: Como mencionamos anteriormente, torna-se premente o emprego e familiaridade com um sistema representacional e notacional particular. Nesse casos, o professor deve estimular a manipulação de representações do tipo $Q_{n}=\left(f_{n}+f_{n+1} i+f_{n+2} j+f_{n+3} \mathrm{k}\right)$ ou ainda a identificação $Q_{n}=\left(f_{n}, f_{n+1}, f_{n+2}, f_{n+3}\right)$. Apesar de que, acentuamos uma representação matricial possível, como assim indica Halici (2012, p. 323),

$$
Q_{n}=\left(f_{n}, f_{n+1}, f_{n+2}, f_{n+3}\right) \rightarrow\left(\begin{array}{cc}
f_{n}+f_{n+1} i & -f_{n+2}-f_{n+3} i \\
f_{n+2}-f_{n+3} i & f_{n}-f_{n+1} i
\end{array}\right)
$$

definida por. E, analogamente a base quadrimensional para os quaternions $\{1, i, j, k\}$, podemos decompor:

$\left(\begin{array}{cc}f_{n}+f_{n+1} i & -f_{n+2}-f_{n+3} i \\ f_{n+2}-f_{n+3} i & f_{n}-f_{n+1} i\end{array}\right)=f_{n} E+f_{n+1} I+f_{n+2} J+f_{n+3} K$ , aonde designamos a base quadrimensional seguinte $E=\left(\begin{array}{ll}1 & 0 \\ 0 & 1\end{array}\right), I=\left(\begin{array}{cc}i & 0 \\ 0 & -i\end{array}\right), J=\left(\begin{array}{cc}0 & -1 \\ 1 & 0\end{array}\right), K=\left(\begin{array}{cc}0 & -i \\ -i & 0\end{array}\right)$.

Figura 3. Hamilton (1853, p, 98) comenta a representação matricial para os quaternions

inferred that it must be true in general. C. S. Peirce (1881) expressed a
guaternion as a matrix thus $w+a x+\beta y+\gamma z$ is equivalent to
\[ \left(\begin{array}{rr}w+i x & y-i z \\ -y-i z & w-i x\end{array}\right) \]
i.e. $w\left(\begin{array}{ll}1 & 0 \\ 0 & 1\end{array}\right)+x\left(\begin{array}{rr}i & 0 \\ 0 & -i\end{array}\right)+y\left(\begin{array}{rr}0 & 1 \\ -1 & 0\end{array}\right)+z\left(\begin{array}{ll}0 & i \\ i & 0\end{array}\right)$. 
Apesar de comentada em Halici (2012), este sistema notacional foi discutido, de modo pioneiro, por Hamilton (1853, p. 98) e, na figura 3, poderemos suscitar uma discussão em torno da sua adaptação ao nosso caso de interesse maior. Assim, as relações algébricas do tipo $f_{n+2}=f_{n+1}+f_{n}$ e $f_{-n}=f_{-(n+1)}+f_{-(n+2)}$, devem estimular a verificação das propriedades correlatas para os quaternions.

Situação de validação: Ora, com base nos dados obtidos as fases anteriores, a seguinte igualdade $Q_{n}+Q_{n+1}=Q_{n+2}$ , bem como $Q_{-n}=Q_{-(n+1)}+Q_{-(n+2)}$ podem ser verificadas. No primeiro caso, os alunos são estimulados a desenvolver a $Q_{n}+Q_{n+1}=\left(f_{n}+i f_{n+1}+j f_{n+2}+k f_{n+3}\right)+\left(f_{n+1}+i f_{n+2}+j f_{n+3}+k f_{n+4}\right)$ seguinte expressão e, em seguida, agrupando termoa termo, vem que: $Q_{n}+Q_{n+1}=\left(f_{n}+f_{n+1}\right)+i\left(f_{n+1}+f_{n+2}\right)+j\left(f_{n+2}+f_{n+3}\right)$ $+k\left(f_{n+3}+f_{n+4}\right)=\left(f_{n+2}+i f_{n+3}+j f_{n+4}+k f_{n+5}\right)=Q_{n+2}$ E, no segundo, usaremos a seguinte recorrência $f_{-n}=f_{-(n+1)}+f_{-(n+2)}$. Assim, notamos que: $Q_{-(n+1)}+Q_{-(n+2)}=$ $=\left(f_{-(n+1)}+f_{-(n+2)} i+f_{-(n+3)} j+f_{-(n+4)} \mathrm{k}\right)+\left(f_{-(n+2)}+f_{-(n+3)} i+f_{-(n+4)} j+f_{-(n+5)} \mathrm{k}\right)=$ $=\left(f_{-(n+1)}+f_{-(n+2)}\right)+\left(f_{-(n+2)}+f_{-(n+3)}\right) i+\left(f_{-(n+3)}+f_{-(n+4)}\right) j+\left(f_{-(n+4)}+f_{-(n+5)}\right) \mathrm{k}=$ $=f_{-n}+f_{-(n+1)} i+f_{-(n+2)} j+f_{-(n+3)} \mathrm{k}$. Assim, vemos que $Q_{-n}=Q_{-(n+1)}+Q_{-(n+2)}, \forall n \in I N$.

Situação de institucionalização: $\mathrm{Na}$ última fase, o professor apresenta o seguinte teorema. Mais uma vez, temos a oportunidade de fazer incorporar ao patrimônio de conhecimentos do grupo um conhecimento acerca de um teorema recentemente discuto no âmbito da pesquisa em Matemática Pura. Dito isto, apresentaremos uma propriedade apresentada por Akyigit; Kösal \& Tosun (2014) como um teorema recentemente discutido na literatura.

Teorema: Para os quaternions de Fibonacci, verificamos a seguinte relação de recorrência $Q_{n+2}=Q_{n+1}+Q_{n}, n \geq 0$. (Akyigit; Kösal \& Tosun, 2014).

$\mathrm{Na}$ última situação, abordaremos a noção da forma generalizada de uma quaternion de Fibonacci.

Situação problema IV: Num artigo cientifico da década de 60, o autor Horadam (1961), definiu a seguinte sequência $H_{n}=H_{n-1}+H_{n-2}, n \geq 3$, com os valores iniciais $H_{1}=p, H_{2}=p+q$, para os inteiros quaisquer $p, q$. A mesma conhecida por Sequência Generalizada de Fibonacci - SGF. Em seguida, Horadam definiu os elementos $P_{n}=H_{n}+H_{n+1} i+H_{n+2} j+H_{n+3} k$. O referido conjunto representa os Quaternions Generalizados de Fibonacci-QGF que herdam, de algum modo, as propriedades da SGF. Decida se as propriedades estudadas nas situações anteriores são válidas para tal definição.

Comentários: Vale observar a exploração da Sequência Generalizada de Fibonacci proporciona a constatação da seguinte lista $\{p, p+q, 2 p+q, 3 p+2 q, 5 p+3 q, \ldots\}$.
Ademais, os estudantes devem ser estimulados a verificar que $H_{n+1}=f_{n+1} p+f_{n} q$, com $n \geq 0$ e, ainda, a fórmula correspondente de Binnet (como indicada na situação II), para a SGF.

Situação de ação: De modo análogo ao que foi abordado nas situações anteriores, os estudantes devem notar os seguintes valores particulares na sequência: $P_{1}=H_{1}+H_{2} i+H_{3} j+H_{4} k=p+(p+q) i+(2 p+q) j+(3 p+2 q) k$

$$
\begin{aligned}
& P_{2}= H_{2}+H_{3} i+H_{4} j+H_{5} k= \\
& P_{3}=H_{3}+H_{4} i+H_{5} j+H_{6} k=(2 p+q)+ \\
&=(p+q)+(2 p+q) i+(3 p+2 q) j+(5 p+3 q) k \\
&, \quad+(3 p+2 q) \mathrm{j}+(5 \mathrm{p}+3 \mathrm{q}) \mathrm{k}
\end{aligned}
$$

$$
P_{4}=H_{4}+H_{5} i+H_{6} j+H_{7} k=(3 p+2 q)+(5 p+3 q) i+
$$

$+(8 p+5 q) j+(11 p+8 q) k$, etc.. Ademais, a partir da relação de recorrência $H_{n}=H_{n-1}+H_{n-2}$ , podem verificar por indução que $H_{2}=f_{2} p+f_{1} q$ , $H_{2}=f_{3} p+f_{2} q$ e, ao assumirem que $H_{n}=f_{n} p+f_{n-1} q \therefore H_{n+1}=H_{n}+H_{n-1}=\left(f_{n} p+f_{n-1} q\right)+\left(f_{n-1} p+f_{n-2} q\right)=$

$=f_{n} p+f_{n-1} p+f_{n-1} q+f_{n-2} q=\left(f_{n}+f_{n-1}\right) p+\left(f_{n-1}+f_{n-2}\right) q$ , portanto, devem encontrar que $H_{n+1}=f_{n+1} p+f_{n} q$, tendo em vista que $f_{n+1}=f_{n}+f_{n-1}$.

Situação de formulação: $\mathrm{Na}$ fase atual os alunos devem investigar que propriedades dos Quaternions Generalizados de Fibonacci são herdadas da SGF e, por sua vez, se mostram ainda vinculadas com os números da Sequência de Fibonacci.

Assim, devem estudar, também, o seguinte comportamento para índices inteiros negativos, quando avaliarem $H_{-(n+1)}=f_{-(n+1)} p+f_{-n} q=(-1)^{n+2} f_{n+1} \cdot p+(-1)^{n+1} f_{n} \cdot q=$ $=(-1)^{n+1}\left(-f_{n+1} \cdot p+f_{n} \cdot q\right)$

E, a partir dessa relação, devem determinar $P_{-n}=H_{-n}+H_{-(n+1)} i+H_{-(n+2)} j+H_{-(n+3)} k=(-1)^{n}\left(-f_{n} \cdot p+f_{n-1} \cdot q\right)+\left((-1)^{n+1}\left(-f_{n+1} \cdot p+f_{n} \cdot q\right)\right) i+$ $+\left((-1)^{n+2}\left(-f_{n+2} \cdot p+f_{n+1} \cdot q\right)\right) j+\left((-1)^{n+3}\left(-f_{n+3} \cdot p+f_{n+2} \cdot q\right)\right) k=(-1)^{n+1} f_{n} \cdot p+(-1)^{n} f_{n-1} \cdot q+$ $+(-1)^{n+2} f_{n+1} \cdot p i+(-1)^{n+1} f_{n} \cdot q i+(-1)^{n+3} f_{n+2} \cdot p j+(-1)^{n+2} f_{n+1} \cdot q j+(-1)^{n+4} f_{n+3} \cdot p k+(-1)^{n+3} f_{n+2} \cdot q k$

Em seguida, agrupando os elementos acima, devemos determinar que:

$P_{-n}=(-1)^{n+1}\left(f_{n}-f_{n+1} \cdot i+f_{n+2} \cdot j-f_{n+3} \cdot k\right) \cdot p+(-1)^{n}\left(f_{n-1}-f_{n} \cdot i+f_{n+1} \cdot j-f_{n+2} \cdot k\right) \cdot q$ .Podemos observar ainda que, sendo válida a igualdade

$$
\begin{aligned}
& H_{n+1}=f_{n+1} p+f_{n} q=p\left(\frac{\alpha^{n+1}-\beta^{n+1}}{\alpha-\beta}\right)+q\left(\frac{\alpha^{n}-\beta^{n}}{\alpha-\beta}\right)= \\
& H_{n+1}=f_{n+1} p+f_{n} q \text { e, dessa forma, podem substituir } \\
& =\frac{p \alpha^{n+1}-p \beta^{n+1}+q \alpha^{n}-q \beta^{n}}{\alpha-\beta}=\frac{\alpha^{n} \cdot(p \alpha+q)-\beta^{n} \cdot(\mathrm{p} \beta+q)}{\alpha-\beta}
\end{aligned}
$$

Dessa forma, tomando $\mathrm{a}=(p \alpha+q)$ e $\mathrm{b}=(\mathrm{p} \beta+q)$ , podemos estabelecer a fórmpula de Binnet, indicada agora por $H_{n+1}=\frac{a \cdot \chi^{-}-b^{\circ} \beta^{2}}{\alpha-\beta}$, que deve estimular os 
estudantes quanto ao grau de semelhança com a fórmula de Binnet, no caso da sequência de Fibonacci original.

Finalmente, derivar a seguinte relação $P_{n}=H_{n}+H_{n+1} i+H_{n+2} j+H_{n+3} k=$

$=\left(f_{n} p+f_{n-1} q\right)+\left(f_{n+1} p+f_{n} q\right) \cdot i+\left(f_{n+2} p+f_{n+3} q\right) \cdot j+\left(f_{n+3} p+f_{n+4} q\right) \cdot k=$

$=f_{n} p+f_{n+1} p \cdot i+f_{n+2} p \cdot j+f_{n+3} p \cdot k+f_{n-1} q+f_{n} q \cdot i+f_{n+3} q \cdot j+f_{n+4} q \cdot k=$

$=p\left(f_{n}+f_{n+1} \cdot i+f_{n+2} \cdot j+f_{n+3} \cdot k\right)+q\left(f_{n-1}+f_{n} \cdot i+f_{n+3} \cdot j+f_{n+4} \cdot k\right)=p Q_{n}+q Q_{n-1}$

Assim, obtivemos que $P_{n}=\mathrm{p} \cdot \mathrm{Q}_{n}+q \cdot Q_{n-1}$. Por

$$
Q_{n}=\frac{\alpha^{n} \cdot \bar{\alpha}-\beta^{n} \cdot \bar{\beta}}{\alpha-\beta}
$$

isso, pela situação anterior, sabendo que, substituiremos:

$P_{n}=\mathrm{p} \cdot \mathrm{Q}_{n}+q \cdot Q_{n-1}=p\left(\frac{\alpha^{n} \cdot \bar{\alpha}-\beta^{n} \cdot \bar{\beta}}{\alpha-\beta}\right)+q\left(\frac{\alpha^{n-1} \cdot \bar{\alpha}-\beta^{n-1} \cdot \bar{\beta}}{\alpha-\beta}\right)$

$=\frac{p \alpha^{n} \cdot \bar{\alpha}+q \alpha^{n-1} \cdot \bar{\alpha}-\left(p \beta^{n} \cdot \bar{\beta}+q \beta^{n-1} \cdot \bar{\beta}\right)}{\alpha-\beta}=\frac{\alpha^{n-1} \cdot \bar{\alpha}(\alpha p+q)-\beta^{n-1} \cdot \bar{\beta}(\beta p+q)}{\alpha-\beta}$

$P_{n}=\frac{\alpha^{n-1} \cdot \bar{\alpha}(\alpha p+q)-\beta^{n-1} \cdot \bar{\beta}(\beta p+q)}{\alpha-\beta}$

Por conseguinte, derivamos a igualdade que corresponde a fórmula de Binnet para os números da SGQF.

Situação de validação: Desde que valem as relações $P_{n}=H_{n}+i H_{n+1}+j H_{n+2}+k H_{n+3}$ $P_{n+1}=H_{n+1}+i H_{n+2}+j H_{n+3}^{n}+k H_{n+4}$ e, por conseguinte, vemos $P_{n}^{n+1}+P_{n+1}=\left(H_{n}+i H_{n+1}^{n+3}+j H_{n+2}^{n+4}+k H_{n+3}\right)+$

$+\left(H_{n+1}+i H_{n+2}+j H_{n+3}+k H_{n+4}\right)$ e, repetindo um argumento já visto, resulta na igualdade $P_{n}+P_{n+1}=P_{n+2}$

- E, ainda, empregando a relação abordada na situação anterior, que indicamos por $H_{-n}=H_{-(n+1)}+H_{-(n+2)}$, devem encontrar também $P_{-n}=P_{-(n+1)}+P_{-(n+2)}$, para todo índice inteiro. Com efeito, vejamos o desenvolvimento de $P_{-(n+1)}+P_{-(n+2)}=$

$=H_{-(n+1)}+i H_{-(n+2)}+j H_{-(n+3)}+k H_{-(n+4)}+H_{-(n+2)}+i H_{-(n+3)}+j H_{-(n+4)}+k H_{-(n+5)}=$

$=H_{-(n+1)}+H_{-(n+2)}+i\left(H_{-(n+2)}+H_{-(n+3)}\right)+j\left(H_{-(n+3)}+H_{-(n+4)}\right)+k\left(H_{-(n+4)}+H_{-(n+5)}\right)=$

$=H_{-n}+i H_{-(\mathrm{n}+1)}+j H_{-(n+2)}+k H_{-(n+3)}=P_{-n}$

, para todo índice inteiro.

Desse modo, semelhante ao caso estudado na situação III, vale a relação de recorrência para o caso da generalização da noção de Quaternion Generalizado de Fibonacci - QGF.

Situação de institucionalização: Com origem nas nos resultados anteriores, enunciaremos um teorema que adquirirá o status de conhecimento a ser incorporado ao patrimônio dos saberes matemáticos dos estudantes.

Teorema: A fórmula de Binnet, para os quaternions generalizados de Fibonacci, vale:

$$
\begin{aligned}
& P_{n}=\left\{\begin{array}{l}
\frac{\alpha^{n-1} \cdot \bar{\alpha}(\alpha p+q)-\beta^{n-1} \cdot \bar{\beta}(\beta p+q)}{\alpha-\beta}, \mathrm{n} \geq 0 \\
(-1)^{n} \frac{\alpha^{n-1} \cdot \bar{\alpha}(\alpha p+q)-\beta^{n-1} \cdot \bar{\beta}(\beta p+q)}{\alpha-\beta}, n<0
\end{array}\right. \\
& , \text { aonde } \alpha, \beta=\frac{1 \pm \sqrt{5}}{2}
\end{aligned}
$$

Demonstração: Vamos usar a fórmula

$P_{n}=\frac{\alpha^{n-1} \cdot \bar{\alpha}(\alpha p+q)-\beta^{n-1} \cdot \bar{\beta}(\beta p+q)}{\alpha-\beta}$

obtida anteriormente que indicamos por .Veja

$P_{-n}=\frac{\alpha^{-(n-1)} \cdot \bar{\alpha}(\alpha p+q)-\beta^{-(n-1)} \cdot \bar{\beta}(\beta p+q)}{\alpha-\beta}=$

$=\frac{\alpha^{-n} \cdot \alpha \cdot \alpha(\alpha p+q)-\beta^{-n} \cdot \beta \cdot \bar{\beta}(\beta p+q)}{\alpha-\beta}=\frac{\left(\frac{1}{\alpha}\right)^{n} \cdot \alpha \cdot \alpha(\alpha p+q)-\left(\frac{1}{\beta}\right)^{n} \cdot \beta \cdot \bar{\beta}(\beta p+q)}{\alpha-\beta}=$

$=\frac{(-\beta)^{n} \cdot \alpha \cdot \bar{\alpha}(\alpha p+q)-(-\alpha)^{n} \cdot \beta \cdot \bar{\beta}(\beta p+q)}{\alpha-\beta}=(-1)^{n} \frac{\beta^{n} \cdot \alpha \cdot \alpha(\alpha p+q)-\alpha^{n} \cdot \beta \cdot \bar{\beta}(\beta p+q)}{\alpha-\beta}=$ $=(-1)^{n} \frac{\beta^{n-1} \cdot(\beta \alpha) \cdot \alpha(\alpha p+q)-\alpha^{n-1} \cdot(\alpha \beta) \cdot \bar{\beta}(\beta p+q)}{\alpha-\beta}=(-1)^{n+1} \frac{\beta^{n-1} \cdot \bar{\alpha}(\alpha p+q)-\alpha^{n-1} \cdot \bar{\beta}(\beta p+q)}{\alpha-\beta}$

$P_{-n}=(-1)^{n+1} \frac{\beta^{n-1} \cdot \bar{\alpha}(\alpha p+q)-\alpha^{n-1} \cdot \bar{\beta}(\beta p+q)}{\alpha-\beta}$

Por fim, de modo pormenorizado, escrevemos

$=(-1)^{n+1} \frac{\beta^{n-1}(\alpha p+q)\left(1+i \alpha+j \alpha^{2}+k \alpha^{3}\right)-\alpha^{n-1}(\beta p+q)\left(1+i \beta+j \beta^{2}+k \beta^{3}\right)}{\alpha-\beta}$

. Ora, com isto, evidenciamos que $P_{-n}=(-1)^{n+1} P_{n}, n \in I N$

Dessa forma, com origem num conjunto de informações, recorrentemente desconsideradas numa cultura de graduação em Matemática, estabelecemos os seguintes resultados mais representativos do percurso investigativo proposto e que consubstanciam os objetivos propostos ao decurso do trabalho (itens (a), (b) e (c)).

- Semelhantemente ao caso do modelo de Fibonacci, para os quaternions de Fibonacci valem as relações de recursividade

e

$$
\left\{\begin{array}{l}
Q_{n+2}=Q_{n+1}+Q_{n}, n \geq 0 \\
Q_{-n}=Q_{-(n+1)}+Q_{-(\mathrm{n}+2)}, n \geq 0
\end{array}\right.
$$

$$
\left\{\begin{array}{l}
P_{n}+P_{n+1}=P_{n+2}, n \geq 0 \\
P_{-n}=P_{-(n+1)}+P_{-(n+2)}
\end{array}\right.
$$

Tal propriedade, semelhantemente ao caso da Sequência de Fibonacci, envolve a extensão dos índices dos quaternions (generalizados) de Fibonacci;

- Semelhantemente ao caso do modelo de Fibonacci, para a determinação de sua fórmula explícita, para os quaternions de Fibonacci vale a fórmula de Binnet, para índices inteiros 
quaisquer, da forma:

$\left\{\begin{array}{l}\bar{\alpha}=1+i \alpha+j \alpha^{2}+k \alpha^{3} \\ \bar{\beta}=1+i \beta+j \beta^{2}+k \beta^{3} \\ \overline{\bar{\alpha}}=1-i \alpha+j \alpha^{2}-k \alpha^{3} \\ \overline{\bar{\beta}}=1-i \beta+j \beta^{2}-k \beta^{3}\end{array} Q_{n}=\left\{\begin{array}{l}\frac{\alpha^{n} \cdot \bar{\alpha}-\beta^{n} \cdot \bar{\beta}}{\alpha-\beta}, n \geq 0 \\ \frac{\alpha^{n} \cdot \bar{\alpha}-\beta^{n} \cdot \overline{\bar{\beta}}}{\alpha-\beta}, n<0\end{array}\right.\right.$ , aonde ;

- Semelhantemente ao caso do modelo de Fibonacci, para os Quaternions Generalizados de Fibonacci - QGF vale

$$
P_{n}=\left\{\begin{array}{l}
\frac{\alpha^{n}(\alpha p+q) \cdot \bar{\alpha}-\beta^{n}(\beta p+q) \cdot \bar{\beta}}{\alpha^{2}+1}, \mathrm{n} \geq 0 \\
(-1)^{n} \mathrm{P}_{n}, n<0
\end{array}\right.
$$

a fórmula de Binnet, para índices inteiros quaisquer, da forma, aonde $\alpha, \beta=\frac{1 \pm \sqrt{5}}{2}$.

- Semelhantemente ao caso do modelo de SGF, para a SGQF admite a fórmula explícita dos termos definidos com índices inteiros quaisquer, do tipo $P_{-n}=(-1)^{n+1}\left(f_{n}-f_{n+1} \cdot i+f_{n+2} \cdot j-f_{n+3} \cdot k\right) \cdot p+(-1)^{n}\left(f_{n-1}-f_{n} \cdot i+f_{n+1} \cdot j-(-1)^{n+3} f_{n+2} \cdot k\right) \cdot q$

- Por intermédio do enunciado das quatro situações problema, buscamos transferir a responsabilidade (devolução) da tarefa acerca da validade ou possibilidade de obtenção de propriedades ensejadas.

As demonstrações dos resultados anteriores devem ser submetidas "a um processo de constante crítica e revalidação" (Davis \& Reush, 1981, p. 142), por parte do grupo. Dessa forma, com arrimo dos resultados predecessores, assinalamos uma perspectiva que se coaduna com nosso ponto de vista. Acentuamos que no sistema clássico de ensino, o professor não controla o meio no qual a situação é apresentada, nem os conhecimentos elaborados pelos estudantes. Ou o aluno aprende sempre em situação, e tal situação é construida deliberadamente ou não pelo professor. Os exemplos, os exercícios encontrados, a ordem da aparição das noções, formam a base do meio para a aprendizagem e, a partir da mesma, os conhecimentos deverão se formar, ao mesmo tempo precisos ou errôneos, sem que o controle total do professor seja possível” (Margolinas, 1995, p. 344).

Para concluir, as ponderações de Margolinas (1995) no excerto anterior se aproximam com o estilo de abordagem que propugnamos para a investigação histórica, no âmbito da formação de professores.

A ordem, a gradação progressiva abstrativa de aparecimento de noções (e definições) intimamente relacionadas com o modelo de Fibonacci, pode ser apreciado, por exemplo, pelo estabelecimento não linear, por vezes avançado, e por vezes letárgico, de definições matemáticas formais (Davis \& Hersh, 1981, p. 141). Tal movimento dialético assume papel imprescindível para o crescimento do edifício da Matemática.

Isso posto, apresentamos uma última definição formulada no trabalho de Kecilioglu \& Akkus (2013, p. 3) que confirma o interesse pela generalização da noção aqui discutida.
Definição 8:Para $n \geq 0$, on-ésimooctônio deFibonacci, será definido por $Q_{n}=\sum_{s=0} f_{n+s} e_{s}$, respectivamente. E o conjunto $\left\{e_{0}, e_{1}, e_{2}, e_{3}, e_{4}, e_{5}, e_{6}, e_{7}\right\}$ constitui uma base canônica dos octônios. (KECILIOGLU \& AKKUS, 2013).

Recorrendo ao ponto de vista de Seródio (2008, p. $156)$, evidenciamos a identificação acima do conjunto $\left\{e_{0}, e_{1}, e_{2}, e_{3}, e_{4}, e_{5}, e_{6}, e_{7}\right\}$, com a seguinte base $\{1, i, j, i j, k, i k, j k, i j k\}$.

Nas figuras 4 e 5 divisamos duas representações possíveis e, acentuamos que, ao lado direito, observamos a representação mnemônica conhecida como "plano de Fano" ou um plano projetivo com sete pontos (Baez, 2001, p. 152; Killgore, 2015, p. 5), que possibilita determinamos as operações formais derivadas e condicionadas com o conjunto $\{1, i, j, i j, k, i k, j k, i j k\}$, em termos dos elementos da base dos quaternions, que comentamos nas situações passadas. Observamos o caráter mnemônico tendo em vista a aplicação de determinadas regras operacionais que regem o trato analítico-procedural dos quaternions e dos octônios (de Fibonacci). (Figura 4, ao lado direito).

Figura 4 - Kecilioglu \& Akkus (2013, p. 2) indicam uma tabela de multiplicação dos octônios e, ao lado direito, Wang \& Ren (2014, p. 165) fornecem uma interpretação mnemônica para os respectivos produtos dos elementos da base canônica dos octônions.

\begin{tabular}{|r||r|r|r|r|r|r|r|r|r|}
\hline & 1 & $\mathrm{e}_{1}$ & $\mathrm{e}_{2}$ & $\mathrm{e}_{3}$ & $\mathrm{e}_{4}$ & $\mathrm{e}_{5}$ & $\mathrm{e}_{6}$ & $\mathrm{e}_{7}$ & \\
\hline \hline 1 & 1 & $\mathrm{e}_{1}$ & $\mathrm{e}_{2}$ & $\mathrm{e}_{3}$ & $\mathrm{e}_{4}$ & $\mathrm{e}_{5}$ & $\mathrm{e}_{6}$ & $\mathrm{e}_{7}$ & \\
\hline $\mathrm{e}_{1}$ & $\mathrm{e}_{1}$ & -1 & $\mathrm{e}_{3}$ & $-\mathrm{e}_{2}$ & $\mathrm{e}_{5}$ & $-\mathrm{e}_{4}$ & $-\mathrm{e}_{7}$ & $\mathrm{e}_{6}$ & \\
\hline $\mathrm{e}_{2}$ & $\mathrm{e}_{2}$ & $-\mathrm{e}_{3}$ & -1 & $\mathrm{e}_{1}$ & $\mathrm{e}_{6}$ & $\mathrm{e}_{7}$ & $-\mathrm{e}_{4}$ & $-\mathrm{e}_{5}$ \\
\hline $\mathrm{e}_{3}$ & $\mathrm{e}_{3}$ & $\mathrm{e}_{2}$ & $-\mathrm{e}_{1}$ & -1 & $\mathrm{e}_{7}$ & $-\mathrm{e}_{6}$ & $\mathrm{e}_{5}$ & $-\mathrm{e}_{4}$ & \\
\hline $\mathrm{e}_{4}$ & $\mathrm{e}_{4}$ & $-\mathrm{e}_{5}$ & $-\mathrm{e}_{6}$ & $-\mathrm{e}_{7}$ & -1 & $\mathrm{e}_{1}$ & $\mathrm{e}_{2}$ & $\mathrm{e}_{3}$ & \\
\hline $\mathrm{e}_{5}$ & $\mathrm{e}_{5}$ & $\mathrm{e}_{4}$ & $-\mathrm{e}_{7}$ & $\mathrm{e}_{6}$ & $-\mathrm{e}_{1}$ & -1 & $-\mathrm{e}_{3}$ & $\mathrm{e}_{2}$ & \\
\hline $\mathrm{e}_{6}$ & $\mathrm{e}_{6}$ & $\mathrm{e}_{7}$ & $\mathrm{e}_{4}$ & $-\mathrm{e}_{5}$ & $-\mathrm{e}_{2}$ & $\mathrm{e}_{3}$ & -1 & $-\mathrm{e}_{1}$ & \\
\hline $\mathrm{e}_{7}$ & $\mathrm{e}_{7}$ & $-\mathrm{e}_{6}$ & $\mathrm{e}_{5}$ & $\mathrm{e}_{4}$ & $-\mathrm{e}_{3}$ & $-\mathrm{e}_{2}$ & $\mathrm{e}_{1}$ & -1 & 6 \\
\hline
\end{tabular}

Figura 5 - Seródio (2008, p. 157) considerou operações com os octônios afim de descrever os polinômios octônicos.

\begin{tabular}{|c||c|c|c|c|c|c|c|c|}
\hline$*$ & 1 & $i$ & $j$ & $i j$ & $k$ & $i k$ & $j k$ & $i j k$ \\
\hline \hline 1 & 1 & $i$ & $j$ & $i j$ & $k$ & $i k$ & $j k$ & $i j k$ \\
\hline$i$ & $i$ & -1 & $i j$ & $-j$ & $i k$ & $-k$ & $i j k$ & $-j k$ \\
\hline$j$ & $j$ & $-i j$ & -1 & $i$ & $j k$ & $-i j k$ & $-k$ & $i k$ \\
\hline$i j$ & $i j$ & $j$ & $-i$ & -1 & $-i j k$ & $-j k$ & $i k$ & $k$ \\
\hline$k$ & $k$ & $-i k$ & $-j k$ & $i j k$ & -1 & $i$ & $j$ & $-i j$ \\
\hline$i k$ & $i k$ & $k$ & $i j k$ & $j k$ & $-i$ & -1 & $-i j$ & $-j$ \\
\hline$j k$ & $j k$ & $-i j k$ & $k$ & $-i k$ & $-j$ & $i j$ & -1 & $i$ \\
\hline$i j k$ & $i j k$ & $j k$ & $-i k$ & $-k$ & $i j$ & $j$ & $-i$ & -1 \\
\hline
\end{tabular}


Figura 6 - Oliveira (2018) desenvolveu uma pesquisa com um campo epistémico ampliado sobre a Sequência Generalizada de Fibonacci (SGF)

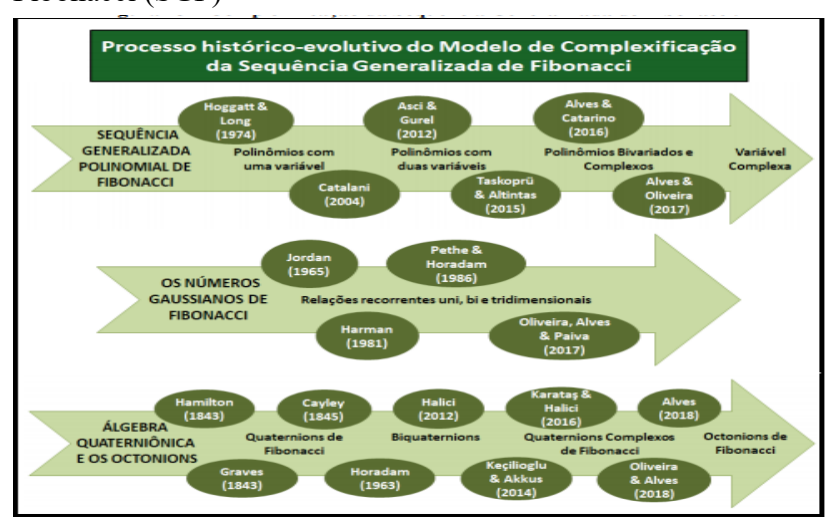

Antes de concluirmos, a evolução progressiva e familiaridade adquirida com o estudo dos quaternions de Fibonacci estimulará, em maior ou em menor substância, a curiosidade ou interesse dos estudantes envolvendo a generalização da noção de quaternion de Fibonacci. Movimento semelhante pode ser observado no caso da pesquisa sobre os quaternions e, posteriormente, dos octônions (Hanson, 2006).

De fato, a definição 8 confirma o ininterrupto interesse por parte dos matemáticos profissionais tendo em vista sua maior descrição em termos de generalidade. Outrossim, com uma atenção cuidadosa, registramos a influência dos trabalhos envolvendo a teoria dos polinômios octônicos (Bernard, 1979; Seródio, 2010) e o estudo de suas propriedades que atuaram no sentido de balizar, confirmar a validez da teoria no âmbito dos quaternions e octônios de Fibonacci. Todavia, o tema octônions de Fibonacci poderá ser um objeto de discussão para a proposição de uma outra Engenharia Didática, diante da inexistência da discussão sobre esta matéria. Para concluir, exibimos na figura 5 acima, Oliveira (2018) apresenta um cenário de desenvolvimento histórico, matemático e epistemológico. O viés histórico, discutido por Oliveira (2018), se caracterizou pelo interesse de gênese das ideias primitivas relacionadas com a sequência de Fibonacci. O componente epistemológico discutido por Oliveira (2018) se consubstancia pelos processos de sistemátização e evolução de ideias e novos modelos especializados, que confirmam um estádio dinâmico de evolução científica

\section{Considerações Finais}

Abordamos no presente trabalho, de natureza teórica, os elementos que assumimos constituir papel essencial na consubstanciação das duas etapas (análise preliminar e análise a priori) tendo em vista uma eventual experimentação em sala de aula, afetada pelo viés investigativo, sistemático e metodológico de uma ED, bem como uma mediação planejada e inspirada nos pressupostos e fundamentos da TSD, cujo caráter de uso em complementaridade se mostra recorrente em vários trabalhos produzidos no âmbito da Didática da Matemática, de vertente francófona (Artigue, 1984; 1989; 2009; 2012; Brousseau, 1986; Robinet, 1983).

Isso posto, os elementos elencados nas análises preliminares, prevista por uma Engenharia Didática, nos permitem perspectivar pontos de vista alvissareiros para o ensino da noção de quaternions de Fibonacci que, a partir de uma formulação indicada, do ponto de vista histórico, por Horadam (1963) (e a produção de várias definições), atraiu o interesse de matemáticos profissionais em trabalhos científicos recentes, todavia, restritos a um ambiente acadêmico restrito (Falcon, 2016; Flaut \& Shpakivskyi, 2013; Halici, 2015; Thongmoon, 2009).

Dessa forma, nas quatro situações-problemas abordadas, balizadas pela perspectiva da TSD, discutimos propriedades e evidenciamos elementos invariantes, na condição em que comparamos o modelo da sequência de Fibonacci com o dos quaternions. E, de modo semelhante ao caso de trabalhos atuais que discutem o emprego da ED, como instrumento teórico-conceitual, capaz de demarcar um conjunto de práticas investigativas de ordem históricas, que concorrem para o aumento dos conhecimentos didáticos e metodológicos sobre um assunto particular e, em nosso caso, não trivial e ainda não discutido sob a égide do ponto de vista aqui apresentado.

Assim, assinalamos que o aumento dimensional e a correspondente investigação por propriedades matemáticas específicas, no que concerne à sua invariância, representa um modus operandi empregado por matemáticos profissionais (Hanson, 2006) e que pode, respeitadas determinadas características, no contexto da investigação histórica e formação de professores, se tornar objeto de reflexão. E, com essa atenção, tendo em vista alcançar os objetivos acentuados no início do trabalho, apresentamos situações didáticas estruturadas e que permitem o estudante um aumento e familiaridade progressiva com os elementos simbolizados ao longo do texto por $Q_{n}=f_{n}+f_{(n+1)} i+f_{(n+2)} j+f_{(n+3)} k$ ou $P_{n}, H_{n}+H_{n+1} i+H_{n+2} j+H_{n+3} k$, para todo inteiro

Por fim, os elementos aqui discutidos de ordem e viés estritamente teórico detêm o potencial de proporcionar uma abordagem distinguida e diferenciada para a noção de quaternions de Fibonacci, que se assemelha, em muito, com alguns trabalhos teóricos da vertente francesa em seu momento histórico e evolutivo de maior visibilidade (Douady \& Douady; 1994; Grenier \& Legrand, 1986).

E, assim, produzimos uma trajetória teórico-conceitual de mediação tanto para esta noção, bem como, para a sua generalização natural, que consiste em considerarmos um conjunto de dimensão 8 (oito), estudado na literatura recente (Halici, 2015; Savin, 2015) como a classe dos octônions de Fibonacci ou octônios complexos e generalizados de $Q_{n}=\sum_{s=0}^{7} f_{n+s} e_{s}=f_{n} e_{0}+f_{n+1} e_{1}+f_{n+2} e_{2}+f_{n+3} e_{3}+f_{n+4} e_{4}+f_{n+5} e_{5}+f_{n+6} e_{6}+f_{n+7} e_{7}$

Fibonacci, denotado pelos matemáticos profissionais como . Todavia, não podemos desconsiderar que, o modelo fundante da álgebra do quaternions, propugnada por Hamilton em 1843, proporciona a solidez dos argumentos aqui discutidos, bem como, o modelo estrutural e fundante da álgebra dos octônions, apresentada por um amigo de Hamilton, o matemático John Thomas Graves (1806 - 1870), em 1844 (2001, p. 1146), 
conferiu a confiança nas operações empregadas séculos depois.

Finalmente, convidamos agora o leitor a comparar a figura mnemônica (ver figura 1) relacionada com a sequência numérica de Fibonacci que, de forma recorrente, costuma ser mencionada por parte dos autores de compêndios especializados de História da Matemática (Huntley, 1970), com ênfase em seus aspectos lúdicos e pitorescos, em detrimento de um componente matemático e estrutural, que ainda preserva um vigor matemático da pesquisa científica, em vários países, (ver figura 6) desta sequência recorrente e que se constitui como um exemplo em constante atualização e não estático e apartado de nosso realidade atual, a ser incorporado, de forma robusta, no repertório de saberes matemáticos e científicos do futuro professor de Matemática.

\section{Agradecimentos}

Agradecemos o suporte no Brasil e o apoio financeiro concedido pelo Conselho Nacional de Desenvolvimento Científico e Tecnológico - CNP para o desenvolvimento dessa pesquisa.

\section{Referências}

Abramovich, S. (2009). Extending Fibonacci numbers to negative subscripts through problem solving. International Journal of Mathematical Education in Science and Technology. 41(6), $836-842$.

Almouloud, A. S. (2007). Fundamentos da Didática da Matemática. São Paulo: Editora UFPR.

Alves, F. R. V. \& Borges Neto, H. (2011). A existência da Sequência de Fibonacci no campo dos Inteiros: uma atividade de investigação apoiada nos pressupostos da Sequência Fedathi. Boletim GEPEM. 1(53). 135 - 140

Alves, Francisco. R. V. (2015a). Sobre a evolução histórica do modelo de Fibonacci: a classe das funções hiperbólicas de Fibonacci. Vydya Educação, 35(1), 1 - 15.

Alves, F. R. V. (2015b). Sequência Generalizada de Fibonacci - SGF e relações com a razão áurea. Boletim Cearense de Educação e História da Matemática - BOCEHM, 2(6), 1 - 5.

Alves, F. R. V. (2016b). Sequência de Pell Generalizada - SGP: aspectos históricos e epistemológicos sobre a evolução de um modelo. Revista THEMA, 13(1), $1-25$.

ALVES, F. R. V. (2016b). Descobrindo definições matemáticas no contexto de investigação histórica: o caso da sequência generalizada de Fibonacci. BOLETIM GEPEM, 69(1), 1 - 5.

Alves, Francisco. R. V. (2016c). Engenharia Didática para a generalização da Sequência de Fibonacci: uma experiência num curso de licenciatura. Educação Matemática Pesquisa. 18(1), $61-93$.

Alves, Francisco. R. V. (2017). Fórmula de de Moivre, ou de Binet ou de Lamé: demonstrações e generalidades sobre a sequência generalizada de Fibonacci - SGF. Revista Brasileira de História da Matemática. 17(33), 1 - 16.

Artigue, M. (1984). Modélisation et Reproductibilité en Didactiques de Mathématiques. Les Cahiers Rouge des Didactiques des Mathematiques, 8(1), p. 1 - 38.

Artigue, M. (1989). Une recherche d'ingènierie didactique sur l'enseignement des Èquations différentielles en premier cycle universitaire. Cahier du Séminaire de Didactique des Maths et de l'Informatique de Grenoble, p. 183-209, IMAG, Grenoble.
Artigue, M. (2009). Didactical design in Mathematics Education. Carl Winslow (eds). NORMA08, Copenhaguen: Sense Publishers, Denmark, 7 - 16.

Artigue, M. (2012). L'éducation mathématiques comme champ de recherché et champ de pratique: resultats et défis. $E M$ TEIA: Revista de Educação Matemática e Tecnológica Iberoamericana, 3(3), $1-18$.

Ávila, G. (2002). O ensino do Cálculo e da Análise. Matemática Universitária. 33(1), p. 83 - 95.

Baez, John. C. (2001). The Octonions. Bullettin American Mathematical Society, 39(1), 145 - 205.

Bernard, Beck. (1979). Sur les équations polynomiales dans les quaternions. In. L'Enseignement Mathématique, 25(1), 193 201.

Brother, Alfred. U. (1965). An introduction to Fibonacci Discovery. Santa Clara: Santa Clara University, California.

Brousseau, Alfred. (1971). Linerar recursion and Fibonacci sequences. Santa Clara: Santa Clara University, California.

Brousseau, G. (1986). Théorisation des phénomènes d'enseignement des Mathématiques (these de doctorat). Bourdeaux: Université Bourdeaux I, $905 \mathrm{f}$.

Brousseau, G. (1988). Le contrat didactique: le milieu. Recherche en Didactiques des Mathematiques, 9(2), p. 309 - 333.

Brousseau, G. (1998). Les obstacles épistémologiques, problèmes et ingénierie didactique. G. In: Brousseau, (org.) Théorie des situations didactiques. Grenoble La Pensée Sauvage, p. 115 -160 .

Buffet. Cécile. Ouvrier. (2003). Construction de definitions/ construction de concept: vers une situation fondamentale pour la construction de définition en mathématriques. Grenoble: Université Joseph Fourier.

Burton, D. (2007). The History of Mathematics: an introduction, New York: McGraw-Hill Science.

Catarino, P. M.; (2015). A note on $\mathrm{h}(\mathrm{x})$ - Fibonacci quaternion polynomials. Chaos, Solitons \& Fractals, 77(4), 1 - 5.

Catarino, P. M. C. (2016). The modified Pell and the Modified k-Pell Quaternions and Octonions, Advances in Applied Clifford Algebra, 26(2), 577 - 590.

Catarino, P. M. C. (2019). On k-Pell hybrid numbers, Journal of Discrete Mathematical Sciences and Cryptography, 22(1), $83-89$.

Chevallard. Y. (1991). La Transposition didactique. Paris: La Pensée Sauvage Édition.

Conway, John. H. \& Smith, Derek. A. (2003). On quaternions and Octonions: their geometry, arithmetic and symmetry. London: A. K. Peters.

Davis, P, J. \& Hersh, R. (1981). L'Univers Mathématique, Paris: Gauthier \& Villars.

Douady, Régine. (1984). Jeux de Cadres et dialetique d'outil-objet dans l'enseignement de Mathématiques - une réalisation dans tout cursus primaire (thése d'État). Paris: Université Paris VII. 262f.

Douady, Régine. (1995a). La ingeniería didáctica y la evolución de su relación con el conocimiento. Gomez, P. (org.) Ingenieria Didactica en Educación Matemática. Bogotá: Grupo Editorial Iberoamericano, p. 1 - 7.

Douady, Régine. (1995b). Nacimiento y desarrollo de la didáctica de las matemáticas en Francia: rol de los IREM. Gomez, P. (org.) Ingenieria Didactica en Educación Matemática. Bogotá: Grupo Editorial Iberoamericano, p. 61 - 97.

Douady, Régine. (2008). Géométrie, graphiques, fonctions au 
collège. Revista Eletrónica de investigación en educación e ciencias. 1(2), p. 1-7.

Douady, A. \& Douady, R. (1994). Changement de cadre a partir de surface minimale. Le Cahier Rouge, n 23, $1-48$.

Eves, H. (2004). Introdução à história da matemática; tradução: Hygino H. Domingues. Campinas, SP: Editora da Unicamp.

Falcon, S. (2016). On the complex k-Fibonacci numbers. Cogent Mathematics, v. 3, n ${ }^{\circ} 1,1-9$.

Flaut, C. \& Shpakivskyi, V. (2013). Real Matrix representations for the Complex Quaternion. Advanced Applied Clifford Algebra, May, 10(2), $657-671$.

Grenier, D. \& Legrand, M. (1986). Un sequence d'enseignement sur $1^{\prime}$ integrale en DEUG a $1^{\circ}$ anée. Le Cahier Rouge, $\mathrm{n}^{\circ} 22$, $1-90$.

Gulberg, J. (1997). Mathematics: From the Birth of Numbers. New York: W. W. Norton \& Company.

Halici, Serpil. \& Karatas, Adnan. (2016). Some Matrix Representations of Fibonacci Quaternions and Octonions. Advanced Clifford Algebra. April, 12(2), p. 123 - 143.

Halici, Serpil. (2015). On Dual Fibonacci Octonions. Advanced Clifford Algebra, April, 25(4), 905 - 914.

Halici, Serpil. (2012a). On Complex Fibonacci quaternions. Advanced Clifford Algebra, June, 23(1), 105 - 112.

Halici, Serpil. (2012b). On Fibonacci Quaternions. Advanced Clifford Algebra, June, 22(2), 321-327.

Hamilton, William, H. (1853). Lectures on Quaternions. Dublin: The Royal Irish Academy.

Hanson, Andrew. J. (2006). Visualizing Quaternions, London: Elsevier.

Harman, C. H. (1981). Complex Fibonaci Numebrs, The Fibonacci Quarterly, 19(1), 82 - 87.

Hoggat, Vernner, E. (1979), Fibonacci and Lucas Numbers, The Fibonacci Association, Houghton Mifflin Company: USA.

Horadam, A. F, (1961). A generalized Fibonacci sequence, $A$. Math. M. 68(5), 455-459.

Horadam. A. F. (1963). Complex Fibonacci Numbers Quaternions, Americal Mathematical Monthly. 70(2), 289 - 291.

Horadam, A. F. (1967). Special Properties of the sequence $W_{n}(a, b ; p, q)$. The Fibonacci Quaterly, 5(5), December, $424-435$.

Horadam, A. F. (1974). Oresme Numbers, The Fibonacci Quarterly, 12(3), 267 - 271.

Iakin, A. L. (1977). Generalized Quaternions with Quaternion Components. The Fibonacci Quarterly, 15(4), December, $225-230$

Huntley, H.E. The divine proportion: a study in mathematical beauty. New York: Dover Publications, 1970.

Iakin, A. L. (1981). Extended Binet Forms for Generalized Quaternions of Higher Order. The Fibonacci Quarterly, 19(5), December, 410 - 414 .

Jeannin, André, R. (1991). Generalized Complex and Lucas functions. The Fibonacci Quarterly, 29(1), February, 13 - 19.

Laborde, C. (1997). Affronter la complexité des situations didátiques d'apprentissage des mathématiques en classe: défis et tentatives. DIDASKALIA, 10(1), p. $97-112$

Killgore, Peter. L. (2015). The Geometry of the Octonionic Multiplication Table. Baccalaureate of Science in Mathematics, Oregon: Oregon State University.
Koshy. T. (2007). Elementary Number Theory and Applications, second edition, Boston: Elselvier.

Krantz, S. G. (2006). An episodic history of mathematics. Mathematical culture through problem solving, New York: Mathematical Association of America.

Margolinas, C. (1995). D'evolution et institutionnalisation: deux aspects antagonistes du rôle du maître. Didactique des disciplines scientifiques et formation des enseignants, Paris: Maison Édition, p. 342-347.

Nurkan, Semra. K. \& Güven, I. Arlans. (2015). On dual Fibonacci quaternions. In: Advanced Applied Clifford Algebra, 25(2), $403-414$

Oliveira, R. R. (2018). Engenharia didática com o tema: relações bidimensionais, tridimenssionais e n-dimensiionais do modelo de Fibonacci. (Dissertação de mestrado em Mestrado em Ensino de Ciências e Matemática, PGECM) - Instituto Federal de Educação Ciencia e Tecnologia - IFCE.

Polatli, E. \& Kesim, S. (2015). A note on Catalan's identity for the k-fibonacci Quaternions. Journal of Integer Sequences, $18(1), 1-4$

Ramirez, J, L. (2015). Some Combinatorial Properties of the k-Fibonacci and the k-Lucas Quaternions. Versita, 23(2), 201 -212 .

Robinet, J. (1983). De L'ingenierie Didactiques. Les Cahiers Blancs. 1(1), p. $1-11$.

Seródio, Rogério. (2008). Construction of Octonionic Polynomials. Advanced Clifford Algebra, 20(1), 155 - 178.

Singh, P. (1985). The So-called Fibonacci Numbers in Ancient and Medieval India, Historia Mathematica, 12(1), 229 - 244.

Singh, Mamta. et all. (2014). Generalized Fibonacci-Lucas Polynomails. International Journal Advanced Mathematics Sciences, 2(1), $81-87$.

Swamy, M. N. S. (1973). On generalized Fibonacci quaternions. The Fibonacci Quarterly, 31(4), November, 315 - 325.

Soykan, Y. (2019). Tibonacci and Tribonacci-Lucas Sedenions, Mathematics, 7(1), 1 - 19. Disponível em: https://www. mdpi.com/2227-7390/7/1/74

Tasci, Dursun \& Yalcin, Feyza. (2013). Complex Fibonacci p-numbers, Communications in Mathematics and Applications, 4(3), 213 - 218.

Thongmoon, M. (2009). New Identities for the Even and Odd Fibonacci and Lucas numbers, International Journal Contemporary Mathematical Sciences. 4(14), 671 - 676.

Vernner, E. \& Hoggat, Jr. (1969). Fibonacci and Lucas Numbers. Santa Clara: Santa Clara University.

Waddill, M. E; Sacks, L. (1967). Another Generalized Fibonacci Sequence. The Fibonacci Quartely. 5(3), October, 209 - 222.

Walton, J. E. \& Horadam, A. F. (1974). Some aspects of Generalized Fibonacci Numbers, The Fibonacci Quarterly, 31(4), October, $241-250$.

Wang, H. \& Ren, G. (2014). Octonion Analysis of Several Variables. Communications in Mathematics and Statistics. June, $164-185$.

Whittaker, E. T. (1945). The Sequence of Ideas in the Discovery of Quaternions. Proceedings of the Royal Irish Academy. Section A: Mathematical and Physical Science. v. 50, 93-98. 